\title{
Performance-Driven Cascade Controller Tuning with Bayesian Optimization
}

\section{Journal Article}

\section{Author(s):}

Khosravi, Mohammad; Behrunani, Varsha; Myszkorowski, Piotr; Smith, Roy (D); Rupenyan-Vasileva, Alisa Bohos (D); Lygeros, John (1)

\section{Publication date:}

2022-01

Permanent link:

https://doi.org/10.3929/ethz-b-000463412

\section{Rights / license:}

In Copyright - Non-Commercial Use Permitted

\section{Originally published in:}

IEEE Transactions on Industrial Electronics 69(1), https://doi.org/10.1109/tie.2021.3050356

\section{Funding acknowledgement:}

180545 - NFS Dependable Ubiquitous Automation (SNF) 


\title{
Performance-Driven Cascade Controller Tuning with Bayesian Optimization
}

\author{
Mohammad Khosravi, Varsha Behrunani, Piotr Myszkorowski, Roy S. Smith, Alisa Rupenyan and John \\ Lygeros
}

\begin{abstract}
We propose a performance-based autotuning method for cascade control systems, where the parameters of a linear axis drive motion controller from two control loops are tuned jointly. Using Bayesian optimization as all parameters are tuned simultaneously, the method is guaranteed to converge asymptotically to the global optimum of the cost. The data-efficiency and performance of the method are studied numerically for several training configurations and compared numerically to those achieved with classical tuning methods and to the exhaustive evaluation of the cost. On the real system, the tracking performance and robustness against disturbances are compared experimentally to nominal tuning. The numerical study and the experimental data both demonstrate that the proposed automated tuning method is efficient in terms of required tuning iterations, robust to disturbances, and results in improved tracking.
\end{abstract}

Index Terms-PID tunining, auto-tuning, Gaussian process, Bayesian optimization

\section{INTRODUCTION}

Routine maintenance of mechatronic systems requires the periodic tuning of PID controllers of linear or rotational drives to counter the gradual decline of performance due to the increase of friction, loosening of mechanical components, or wear. The corresponding PI and PID gains are often set to conservative values to ensure operation for a broad range of loads or mechanical properties, focusing on disturbance rejection. While gain autotuning is in principle possible, existing off-the-shelf autotuning routines often interfere with safety mechanisms in manufacturing systems and are often avoided.

In classical model-based controller design, a model of the plant is derived from first-principle methods or identified using experimental data, and then a controller is designed based on this model [1]. For example, symmetric optimum tuning [2], and its extensions [3] provide relations between the controller gains and the achievable performance in terms of settling time, phase margin, overshoot, and other relevant metrics. Modelbased methods are data-efficient and tuning can be achieved with relatively few iterations. For cascade control systems however, multiple cycles of tuning might be required, and the achieved values of the controller parameters are mostly on the conservative side. Whenever first-principle methods cannot

The authors are with the Automatic Control Laboratory, ETH Zürich. A. Rupenyan is also with inspire AG, 8092 Zürich, Switzerland. (e-mails: \{khosravm, bvarsha, rsmith, ralisa, lygeros\}@control.ee.ethz.ch). P. Myszkorowski is with Sigmatek AG, piotr.myszkorowski@sigmatek.ch. This project has been funded by the Swiss Innovation Agency (Innosuisse), grant Nr. 31695, and by the Swiss National Science Foundation under NCCR Automation. be used, optimization problems arise in obtaining the final controller, both in computation of the model and the controller, through the minimization of prediction error criterion, or criteria based on controller stability and transient smoothness. A controller that best fits the frequency response of the optimal controller is obtained by optimization or order reduction techniques [4]. Performance-based objective functions based on calculating the error signal between the system output and the input reference signal such as e. g. Integral Square Error (ISE), Integral Absolute Error (IAE) or Integral Time Absolute Error (ITAE), are often minimized to tune controller parameters using genetic algorithms [5] or Particle Swarm Optimization [6], and often require a large number of evaluations to ensure that a global optimum is found. Standard methods, such as the Ziegler-Nichols rule [7] or relay tuning with additional heuristics [8], [9] are routinely used for the tuning in practice and will be used here as benchmark results in simulation.

Various data-driven methods for controller design have been proposed, e.g. virtual reference feedback tuning (VRFT) [10], [11], data-driven inversion-based control [12], direct learning of linear parameter varying (LPV) controllers from data [13], data-driven controller tuning in mixed-sensitivity loop-shaping framework. Data-driven tuning has been explored in an iterative learning approach in [14], and also combined with a learned process model in [15]. While they find application, the methods often work under assumptions of linearity or time invariance for the plant or the closed loop system. Iterative learning approaches might require multiple iterations or interventions through external probing signals or specially designed references. Data-driven methods often do not allow for constraints in the input and output variables, and the choice of reference models is also dependent on prior knowledge of the system [16].

Bayesian optimization (BO) is used for hyperparameter tuning in high-dimensional machine learning models, but it has also gained attention in engineering and has been applied e.g., to control of quadrotors combined with learned dynamics [17], optimization of process set-up parameters [18], and collision avoidance [19]. Bayesian data-driven approaches have been also proposed for the tuning of PID controllers and of nonlinear systems [20]-[23]. These approaches are particularly interesting because of the inherent data-efficiency and flexibility of the Bayesian optimization (BO) method. BO in controller tuning, where stability is guaranteed through safe exploration, has been proposed in [20], and applied to robotic applications [24], and in process systems [22]. Constraint BO tuning is proposed in [25]. 


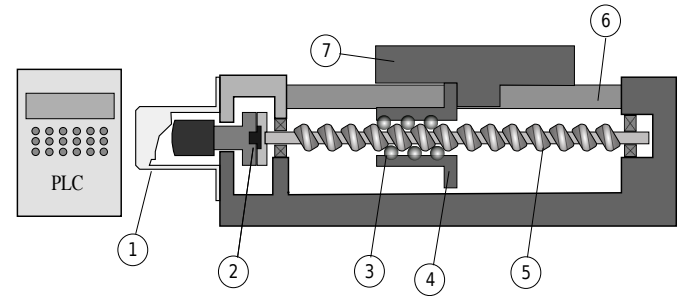

Figure 1: The structure of ball-screw system: (1) DC motor, (2) coupling joint, (3) ball-screw interface, (4) nut, (5) ball-screw shaft, (6) guideway and (7) table (load), following [26].

We propose an automated data-driven tuning method which maximises the performance of a motion system by finding optimal controller parameters. Frequently, tuning of cascade controllers requires multiple iterations switching between controllers at different levels to find an optimum. This paper proposes a model-free autotuning method that is based on the optimization of the combined performance features, extracted from the encoder signals. The proposed method allows considering arbitrary application-oriented performance metrics, defined based on the preferences of the user. Accordingly, the main contributions of this work are: 1) Simultaneous, automated tuning of the parameters of a cascade controller; 2) Numerical demonstration for convergence of the proposed algorithm to the global optimum of the cost, due to the properties of the underlying Bayesian optimization method; 3) Achieving superior performance compared to benchmark tuning methods; 4) Experimental validation for different trajectories, loads, and disturbance rejection, demonstrating the robustness of the proposed approach.

The rest of this paper is organized as follows: Section [II introduces the general problem of performance-driven cascaded controller tuning in linear axis drives. Section III presents the plant model derived from first principles and system identification techniques. Section IV introduces the performancebased approach with numerical results comparing the proposed tuning method with standard tuning methods, also with an evaluation of the performance metrics on a grid. Experimental validation is reported in Section $\mathrm{V}$.

\section{Performance-Based Tuning}

The system of interest is a ball-screw drive, a positioning mechanism used routinely in machining systems. Notable applications of such systems are in the semiconductor industry, in biomedical engineering, and in the photonics and solar technologies. The ball-screw drive components are shown in Figure 1. The AC motor is connected via a coupling joint to a ball-screw shaft fixed to a supporting frame. The shaft carries a nut which converts the rotational motion of the shaft linear motion of the nut through a screw-nut interface. The system is equipped with encoders for measuring the nut position, the rotational speed of the motor and the linear speed of the ballscrew shaft, and a sensor for measuring the motor current. The set voltage of the motor is controlled through a motor drive and a programming logic controller (PLC). Typically, the motor rotates the shaft, the nut moves from initial to final position, remains there for some time and returns to the initial position.

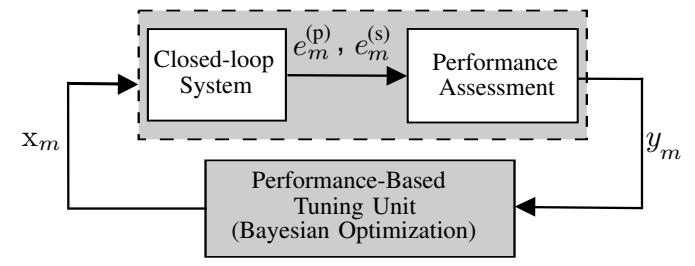

Figure 2: The performance-based controller tuning scheme.

The position and speed of the nut are controlled such that they follow designed ideal reference trajectories (see Figure 4 ).

We introduce a performance metric indicating the quality of tracking, calculated from the position and speed error signals. The error signals and the performance metric are functions of the vector of parameters describing the controller, denoted here by $\mathrm{x}$. Let the performance function be $f: \mathcal{X} \rightarrow \mathbb{R}$ where $\mathcal{X}$ is the space of admissible controller parameters. With respect to controller parameters, $\mathrm{x}$, the performance function does not have a tractable closed-form expression, even when the dynamics of system are known. For a given $\mathrm{x} \in \mathcal{X}$, the value of the performance metric $f(\mathrm{x})$ has a black-box oracle form, and can be obtained experimentally from the position and speed tracking error signals $e^{(\mathrm{p})}$ and $e^{(\mathrm{s})}$, for controller parameters set to $\mathrm{x}$. We propose tuning the controller parameters based on a data-driven procedure of performing a sequence of experiments, collecting data, assessing the corresponding performance, and utilizing Bayesian optimization as a blackbox optimization technique for deriving optimal vector of controller gains (see Figure 2). The estimated performance at each trial is provided to the performance-based tuning unit which is essentially a Bayesian optimization module. This unit models the performance metric using Gaussian process regression (GPR) [27], and finds the optimal parameters based on a suitable Bayesian optimization algorithm as Gaussian process lower confidence bound (GP-LCB) [28].

The proposed tuning approach is performance-based, model-free and data-driven. One of the main advantages of utilizing Bayesian optimization is its potential in explicitly modeling noise which is automatically considered in the uncertainty evaluation without skewing the result [28]. The simultaneous optimization of all parameters guarantees convergence to the global optimum performance, following from the properties of Bayesian optimization, at the cost of more iterations compared to classical approaches.

\section{SYSTEM STRUCTURE AND MOdEL}

\section{A. Mathematical Model of System}

The motor deriving the ball-screw system is a permanentmagnet synchronous motor, with a permanent magnet on the rotor providing the exciting field. Let $R_{\mathrm{s}}$ and $L_{\mathrm{s}}$ respectively denote the resistance and the inductance of the stator. From Kirchhoff's voltage law and the back electromotive force (EMF) which comes from Faraday's law of induction, in the dq-frame we have

$$
\begin{aligned}
& v_{\mathrm{d}}(t)=L_{\mathrm{s}} \frac{\mathrm{d}}{\mathrm{d} t} i_{\mathrm{d}}(t)+R_{\mathrm{s}} i_{\mathrm{d}}(t)-L_{\mathrm{s}} \omega_{\mathrm{m}}(t) i_{\mathrm{q}}(t), \\
& v_{\mathrm{q}}(t)=L_{\mathrm{s}} \frac{\mathrm{d}}{\mathrm{d} t} i_{\mathrm{q}}(t)+R_{\mathrm{s}} i_{\mathrm{q}}(t)+L_{\mathrm{s}} \omega_{\mathrm{m}}(t) i_{\mathrm{d}}(t)+K_{\mathrm{b}} \omega_{\mathrm{m}}(t),
\end{aligned}
$$


where $\left[v_{\mathrm{d}} v_{\mathrm{q}}\right]^{\top}$ and $\left[i_{\mathrm{d}} i_{\mathrm{q}}\right]^{\top}$ are respectively the vectors of stator voltage and stator current in dq-coordinates, $K_{\mathrm{b}}$ is the back EMF constant and $\omega_{\mathrm{m}}$ is the angular velocity of the motor and shaft. The d-component of the current, $i_{\mathrm{d}}$, only influences the component of the magnetic field in the direction of the rotor axis and does not contribute to the torque generation. To minimize losses a PID-controller regulates $v_{\mathrm{d}}(t)$ to compensate the voltage induced by $i_{\mathrm{q}}$ and ensures that $i_{\mathrm{d}}=0$ at all times. Consequently, the resulting dynamics of motor is

$$
\begin{aligned}
& v_{\mathrm{d}}(t)=-L_{\mathrm{s}} \omega_{\mathrm{m}}(t) i_{\mathrm{q}}(t), \\
& v_{\mathrm{q}}(t)=L_{\mathrm{s}} \frac{\mathrm{d}}{\mathrm{d} t} i_{\mathrm{q}}(t)+R_{\mathrm{s}} i_{\mathrm{q}}(t)+K_{\mathrm{b}} \omega_{\mathrm{m}}(t) .
\end{aligned}
$$

The motor develops an electromagnetic torque $\tau_{\mathrm{m}}=K_{\mathrm{t}} i_{\mathrm{q}}$ proportional to the stator q-component of current. One can obtain the transfer function of motor as

$$
M(s):=\frac{\Omega_{\mathrm{m}}(s)}{V_{\mathrm{q}}(s)}=K_{\mathrm{t}}\left(K_{\mathrm{t}} K_{\mathrm{b}}+\left(L_{\mathrm{s}} s+R_{\mathrm{s}}\right)\left(\frac{T_{\mathrm{m}}(s)}{\Omega_{\mathrm{m}}(s)}\right)\right)^{-1},
$$

where $\Omega_{\mathrm{m}}, V_{\mathrm{q}}$ and $T_{\mathrm{m}}$ are the Laplace transform of $\omega_{\mathrm{m}}, v_{\mathrm{q}}$ and $\tau_{\mathrm{m}}$, respectively.

The main limitation on the accuracy of the linear position is due to the first axial mode of the ball-screw system [29], which is determined by the flexibility characteristics of the translating components. This includes the inherent stiffness of the ballscrew and the bearings as well as the interactions between the carriage table, the nut and the ball-screw [26], [29], [30]. The first axial dynamics of the ball-screw servo drive can be modelled using a simplified two degree of freedom massspring-damper system [26]. If we let $J_{\mathrm{m}}, B_{\mathrm{m}}$ and $\theta_{\mathrm{m}}$ be inertia, damping coefficient and angular displacement of the motor, and $J_{1}, B_{l}, \theta_{1}$ and $\omega_{1}$ the corresponding quantities of the load, a torque balance leads to

$$
\begin{aligned}
& J_{\mathrm{m}} \frac{\mathrm{d} \omega_{\mathrm{m}}}{\mathrm{d} t}+B_{\mathrm{m}} \omega_{\mathrm{m}}+B_{\mathrm{ml}}\left(\omega_{\mathrm{m}}-\omega_{\mathrm{l}}\right)+K_{\mathrm{s}}\left(\theta_{\mathrm{m}}-\theta_{\mathrm{l}}\right)=\tau_{\mathrm{m}}, \\
& J_{1} \frac{\mathrm{d} \omega_{\mathrm{l}}}{\mathrm{d} t}+B_{1} \omega_{\mathrm{l}}-B_{\mathrm{ml}}\left(\omega_{\mathrm{m}}-\omega_{\mathrm{l}}\right)-K_{\mathrm{s}}\left(\theta_{\mathrm{m}}-\theta_{\mathrm{l}}\right)=\tau_{\mathrm{l}},
\end{aligned}
$$

where $K_{\mathrm{s}}$ is the total equivalent axial stiffness, $\tau_{1}$ is the torque disturbance of the load and $B_{\mathrm{ml}}$ is the damping coefficient between the coupling and the guides. As $B_{1}$ has a negligible impact on resonance, one can set $B_{1}=0$ [26]. Taking the Laplace transform leads to

$$
\left[\begin{array}{c}
\Theta_{\mathrm{m}}(s) \\
\Theta_{1}(s)
\end{array}\right]=\mathrm{H}(s)^{-1}\left[\begin{array}{c}
T_{\mathrm{m}}(s) \\
T_{1}(s)
\end{array}\right]
$$

where capital letters denote the Laplace transform of the corresponding quantities and $\mathrm{H}(s)$ is defined as

$$
\mathrm{H}(s):=\left[\begin{array}{cc}
J_{\mathrm{m}} s^{2}+\left(B_{\mathrm{m}}+B_{\mathrm{ml}}\right) s+K_{\mathrm{s}} & -B_{\mathrm{ml}} s-K_{\mathrm{s}} \\
-B_{\mathrm{ml}} s-K_{\mathrm{s}} & J_{1} s^{2}+\left(B_{1}+B_{\mathrm{ml}}\right) s+K_{\mathrm{s}}
\end{array}\right] .
$$

Due to the structure of the linear axial system, the torque disturbance of the load comparing to motor torque is negligible. Therefore, to derive the transfer function from motor torque to the angular velocity, we set $T_{1}(s)=0$. Let $D(s)$ denote the determinant of $\mathrm{H}(s)$. To obtain the transfer functions relating the motor to the angular velocities, we define transfer functions

$$
F_{1}(s):=\Omega_{\mathrm{m}}(s) / T_{\mathrm{m}}(s)=\left(J_{1} s^{2}+B_{\mathrm{ml}} s+K_{\mathrm{s}}\right) / D(s),
$$
$F_{2}(s):=\Omega_{\mathrm{l}}(s) / T_{\mathrm{m}}(s)=\left(B_{\mathrm{ml}} s+K_{\mathrm{s}}\right) / D(s)$, and subsequently,

$$
F_{3}(s):=\frac{\Omega_{1}(s)}{\Omega_{\mathrm{m}}(s)}=\frac{F_{2}(s)}{F_{1}(s)}=\frac{B_{\mathrm{ml}} s+K_{\mathrm{s}}}{J_{1} s^{2}+B_{\mathrm{ml}} s+K_{\mathrm{s}}} .
$$

Combining with (2) leads to the transfer function between the voltage applied to the armature and rotational velocity of the load [30]

$$
\begin{aligned}
G(s) & :=\frac{\Omega_{\mathrm{l}}(s)}{V_{\mathrm{q}}(s)}=\frac{\Omega_{\mathrm{m}}(s)}{V_{\mathrm{q}}(s)} \frac{\Omega_{\mathrm{l}}(s)}{\Omega_{\mathrm{m}}(s)}=M(s) F_{3}(s) \\
& =K_{\mathrm{t}}\left(K_{\mathrm{t}} K_{\mathrm{b}}+\left(L_{\mathrm{s}} s+R_{\mathrm{s}}\right) F_{1}(s)^{-1}\right)^{-1} F_{3}(s) .
\end{aligned}
$$

Moreover, if $K_{\mathrm{s}} \gg 1$, one can approximate $F_{1}(s)^{-1}$ by $\left(\left(J_{\mathrm{m}}+J_{1}\right) s+B_{\mathrm{m}}\right)$.

This approximation is valid for the low range of frequencies, typically $\left[0,10^{5}\right] \mathrm{Hz}$, which includes the main frequency range of the operation of the system. The final transfer function is

$$
\begin{gathered}
G(s)=K_{\mathrm{t}}\left(K_{\mathrm{t}} K_{\mathrm{b}}+\left(L_{\mathrm{s}} s+R_{\mathrm{s}}\right)\left(\left(J_{\mathrm{m}}+J_{\mathrm{l}}\right) s+B_{\mathrm{m}}\right)\right)^{-1} \\
\left(\frac{B_{m \mathrm{l}} s+K_{\mathrm{s}}}{J_{\mathrm{l}} s^{2}+B_{m \mathrm{l}} s+K_{\mathrm{s}}}\right) .
\end{gathered}
$$

\section{B. The Control Scheme}

The system is controlled by a PLC that runs a custom-made software package named LASAL. The controller consists of three cascaded loops as shown in Figure 3, where the output signals of each outer loop serve as the reference for the next inner loop. The first block in the axis controller is the interpolation block that receives the trajectory specifications from the user and determines the references for the position and the speed in the system. The interpolation block requires four inputs: position setpoint, speed setpoint, desired acceleration and desired deceleration. Once these inputs are provided, the interpolation block generates a reference speed and position trajectory using the equations of motion. The outer-most control loop is for linear position control. The middle control loop regulates the linear speed. The output of the interpolation block provides these loops with the designed nominal reference for the position and the feed-forward reference for the speed. The motor encoder detects the position of the motor and provides the feedback for both the position and speed control loops.

The controller in the position control loop is a P-controller as $C_{\mathrm{p}}(s)=K_{\mathrm{p}}$, whereas the controller in the speed control loop, is a PI-controller as $C_{\mathrm{s}}(s)=K_{\mathrm{v}}+K_{\mathrm{i}} / s$. One can also introduce $C_{\mathrm{v}}(s)$ in the form of $C_{\mathrm{s}}(s)=K_{\mathrm{v}}\left(1+1 / T_{\mathrm{n}}(s)\right)$ where $T_{\mathrm{n}}$ is the integral time constant of the controller. The speed control loop provides the reference for the current controller, which is the inner-most loop. The feedback in this loop is the

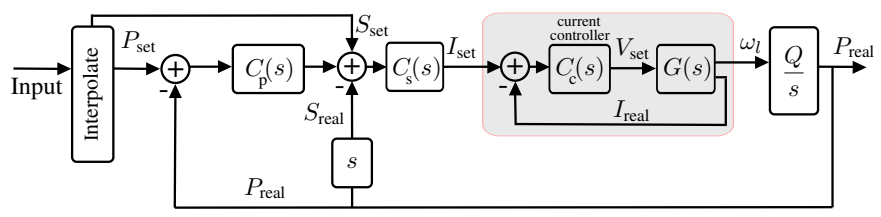

Figure 3: Block diagram of the system 
Table I: Parameters of the current controller and the plant.

\begin{tabular}{lccc}
\hline \multicolumn{2}{c}{ Controller $C(s)$} & \multicolumn{2}{c}{ Plant } \\
\hline Parameter & Value & Parameter & Value \\
\hline$K_{\mathrm{cp}}$ & 60 & $R_{\mathrm{s}}$ & $9.02 \Omega$ \\
$K_{\mathrm{ci}}$ & 1000 & $L_{\mathrm{s}}$ & 0.0187 \\
$K_{\mathrm{cd}}$ & 18 & $K_{\mathrm{t}}$ & $0.515 \mathrm{Vs} \mathrm{rad}^{-1}$ \\
& & $K_{\mathrm{b}}$ & $0.55 \mathrm{Nm} \mathrm{A}^{-1}$ \\
& $J_{\mathrm{m}}$ & $0.27 \times 10^{-4} \mathrm{~kg} \mathrm{~m}^{2}$ \\
& $B_{\mathrm{m}}$ & 0.0074 \\
& $J_{\mathrm{l}}$ & $6.53 \times 10^{-4} \mathrm{~kg} \mathrm{~m}^{2}$ \\
& $B_{\mathrm{ml}}$ & 0.014 \\
& & $K_{\mathrm{s}}$ & $3 \times 10^{7}$ \\
& $Q$ & $1.8 \times 10^{-2} \mathrm{~m}$ \\
& & Max. Speed & 8000 revolutions $/$ minute \\
\hline
\end{tabular}

measured current of the armature. This loop is regulated by a PID-controller block denoted by $C_{\mathrm{c}}(s)=K_{\mathrm{cp}}+K_{\mathrm{ci}} / s+K_{\mathrm{cd}} s$. The output of the controller is the voltage setpoint for the motor given to the motor drive.

Finally, the last block in Figure 3 is not part of the controller but is used to convert the rotational velocity of the ballscrew to linear speed. The factor $Q$ is the linear displacement resulting from one complete rotation of the motor.

The linear axis has three separate modes of operation according to which control loops and parameters are chosen. In position control mode (used in this work), all three feedback loops are active and the position is the critical attribute of the system. In this mode, the controller will try to adhere as close as possible to the position reference even if that entails deviating from the speed trajectory. In the speed control mode (not considered here), the speed trajectory is prioritized, and the position controller deactivated by setting the gain in the position controller to zero, $K_{\mathrm{p}}=0$. Finally, in the current control mode (also not considered here), only the inner-most loop is active and the other controller gains are set to zero.

\section{The Parameters of the Model}

The transfer function of the plant as well as the control loops depend on several parameters. As our work concentrates on tuning the parameters of $C_{\mathrm{p}}(s)$ and $C_{\mathrm{s}}(s)$, it is assumed that the parameters of $C_{\mathrm{c}}(s)$ are given. Values for most of the parameters of the plant are provided or can be calculated from the available data sheets. The only exception is $K_{\mathrm{s}}$. We estimate this parameter experimentally by fitting the step response of the model using least squares. The resulting parameter values are summarised in Table [

\section{Performance-Based Controller Tuning: NUMERICAL INVESTIGATION}

\section{A. Classical Tuning Methods}

The numerical study provided in this section elucidates various aspects and features of the proposed data-driven controller tuning method and compares its performance to widely used tuning techniques. The classical PID tuning approach is the Ziegler-Nichols method, a heuristic designed for disturbance rejection [7], and an automated method where the controller is replaced by a relay and the PID coefficients are estimated based the resulting oscillatory response of the system [8]. Other tuning approaches are also used in practice, where a performance indicator of the system response is minimized, e.g., the integral of time-weighted absolute error (ITAE) [31].

\section{B. Performance-Based Tuning Method}

The performance-based tuning proposed here utilizes Bayesian optimization to tune the controller parameters (see Figure 2). The main ingredient in Bayesian optimization is the cost function, which is composed of a set of metrics capturing the performance requirements of the system. For a linear actuator, in addition to the standard controller tuning metrics such as overshoot and settling time, the position tracking accuracy and the suppression of mechanical vibrations known as oscillation effects are of highest importance.

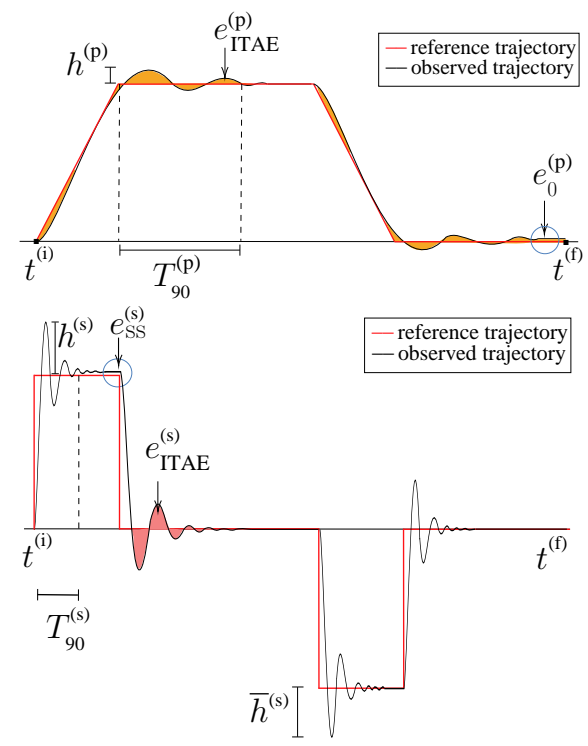

Figure 4: The figure demonstrates various performance metrics for position tracking (top) and speed tracking (bottom). The reference and the observed trajectory are shown respectively by the red line and black line.

Let the vector of controller gains be set to $\mathrm{x}:=$ $\left[K_{\mathrm{p}}, K_{\mathrm{v}}, K_{\mathrm{i}}\right]^{\top}$ and an experiment be performed in time interval $I:=\left[t^{(\mathrm{i})}, t^{(\mathrm{f})}\right]$ with these gains. Once the experiment is completed, the corresponding error signals for position, $e^{(\mathrm{p})}: I \rightarrow \mathbb{R}$, and for speed, $e^{(\mathrm{s})}: I \rightarrow \mathbb{R}$, are measured, and used to calculate the tracking performance metric for $\mathrm{x}$.

For position tracking, the corresponding performance metrics are the overshoot, $h^{(\mathrm{p})}$, the undershoot, $\bar{h}^{(\mathrm{p})}$, the steadystate error, $e_{\mathrm{ss}}^{(\mathrm{p})}$, the settling time, $T_{90}^{(\mathrm{p})}$, and the maximum absolute position error (infinity norm), $\left\|e^{(\mathrm{p})}\right\|_{\infty}$. To minimize oscillations due to the excitation of vibration modes, the integral of the time-weighted absolute value of the error of the position response also defined [31] as $e_{\mathrm{ITAE}}^{(\mathrm{p})}:=\int_{t^{(\mathrm{i})}}^{t^{(\mathrm{f})}}\left(t-t^{(\mathrm{i})}\right)\left|e^{(\mathrm{p})}(t)\right| \mathrm{d} t$, is also included. The position zero error, $e_{0}^{(\mathrm{p})}$, is defined as the error of the system once it returns to zero position (zero) once the motion is completed and it is of high interest, as it 


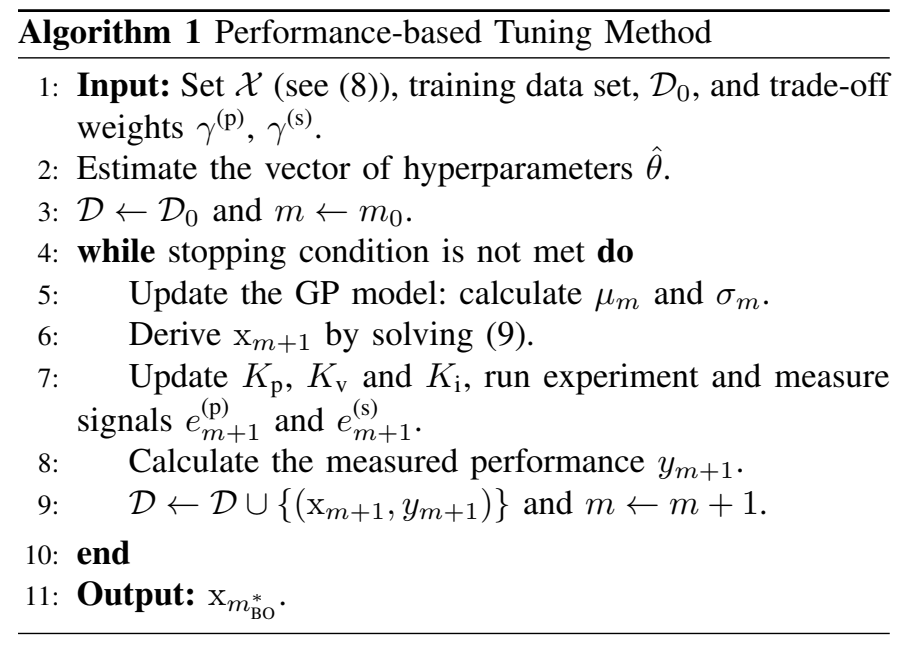

quantifies the oscillations in the system. The same metrics are also used for the speed tracking error and superscript '(s)' is used to denote the corresponding speed metrics.

We take a weighted sum of the individual position metrics, denoted by $F_{i}^{(\mathrm{p})}(\mathrm{x}), i=1, \ldots, N^{(\mathrm{p})}$, with corresponding weights $\gamma_{i}^{(\mathrm{p})}, i=1, \ldots, N^{(\mathrm{p})}$, to generate a combined position metric $f^{(\mathrm{p})}(\mathrm{x})$. Similarly, we generate a combined speed metric, denoted by $f^{(\mathrm{s})}(\mathrm{x})$. In order to generate an overall performance metric for controller tuning, we then take sum of the two quantities as $f(\mathrm{x}):=f^{(\mathrm{p})}(\mathrm{x})+f^{(\mathrm{s})}(\mathrm{x})$.

The values of the different weights used are summarised in Table II. Each of the weights depends on the scale and the importance of corresponding metric and feature and can be selected considering the particular application requirements, e.g. maximization of tracking accuracy in position, minimization of response time, or minimization of oscillations in the system.

A fundamental constraint for tuning the controllers is the stability of the closed-loop system during the tuning procedure. This can be achieved by constraining the controller gains to known ranges guaranteeing this feature. To this end, the feasible set for $\mathrm{x}$, denoted by $\mathcal{X}$, is defined as a safe subset of

$$
\left[K_{\mathrm{p}, \min }, K_{\mathrm{p}, \max }\right] \times\left[K_{\mathrm{v}, \min }, K_{\mathrm{v}, \max }\right] \times\left[K_{\mathrm{i}, \min }, K_{\mathrm{i}, \max }\right],
$$

where $K_{\mathrm{p}, \min }, K_{\mathrm{v}, \min }$ and $K_{\mathrm{i}, \min }$ are the smallest positive values acceptable by the controller, $K_{\mathrm{p}, \max }=4200, K_{\mathrm{v}, \max }=$ 0.5 , and $K_{\mathrm{i}, \max }=900$. In practice, $\mathcal{X}$ is either provided with the system, or estimated in a safe exploration procedure. Here, we have derived $\mathcal{X}$ by a grid computation of the system response for various controller parameters.

\begin{tabular}{lccc}
\hline \multicolumn{2}{c}{ position } & \multicolumn{2}{c}{ speed } \\
\hline$F_{i}^{(\mathrm{p})}$ & $\gamma^{(\mathrm{p})}$ & $F_{i}^{(\mathrm{s})}$ & $\gamma^{(\mathrm{s})}$ \\
\hline$T_{90}^{(\mathrm{p})}$ & $10^{5}$ & $T_{90}^{(\mathrm{s})}$ & $5 \cdot 10^{2}$ \\
$h^{(\mathrm{p})}$ & $10^{2}$ & $h^{(\mathrm{s})}$ & 2 \\
$\left\|e^{(\mathrm{p})}\right\|_{\infty}$ & $10^{3}$ & $\left\|e^{(\mathrm{s})}\right\|_{\infty}$ & $5 \cdot 10^{2}$ \\
& & $e_{\text {ITAE }}^{(\mathrm{s})}$ & $10^{4}$ \\
\hline
\end{tabular}

Table II: Weights in the cost function
Note that $f^{(\mathrm{p})}, f^{(\mathrm{s})}$ and $f$ are functions of controller gains $\mathrm{x}$ in an oracle form. The controller parameters with optimal performance, $\mathrm{x}^{*}$, are the ones inducing minimum cost objective $f$. To find these optimal values, we utilize Bayesian optimization, summarized in Algorithm 1 Starting from feasible set $\mathcal{X}$, we perform a sequence of experiments. In the $m^{\text {th }}$ experiment, a new vector of controller gains $\mathrm{x}_{m}$ is used, and based on the measured error signals, the corresponding performance $f\left(\mathrm{x}_{m}\right)$ is evaluated as $y_{m}$. The first $m_{0}$ experiments are for constructing the initial data set $\mathcal{D}_{0}:=\left\{\left(\mathrm{x}_{m}, y_{m}\right) \mid m=1,2, \ldots, m_{0}\right\}$.

In many practical situations, $\mathcal{D}_{0}$ is already available from previous attempts on tuning the controllers. Otherwise, one can obtain $\mathcal{D}_{0}$ by performing experiments in which the controller parameters are either derived from random perturbations of the nominal gains, or based on a Latin hypercube experimental design [32]. Using GPR and the collected data, we obtain a surrogate function for the performance metric $f$ as a Gaussian process $\mathcal{G P}\left(\mu_{m}, k_{m}\right)$, where $\mu_{m}: \mathcal{X} \rightarrow \mathbb{R}$ is the mean function predicting the value of $f$ at different locations, and $k_{m}: \mathcal{X} \times \mathcal{X} \rightarrow \mathbb{R}$ is the kernel function evaluating the uncertainty in the predictions. Here, we utilize squared exponential kernel with hyperparameters tuned using $\mathcal{D}_{0}$. Since the continuity of $f$ does not change by possible saturation blocks, they can be considered as well in the closed-loop. In this situation, one should utilize kernels which are more suitable for non-differentiable functions such as Matern kernels [27]. The controller gains are updated due to GP-LCB sampling algorithm [28] as

$$
\mathrm{x}_{m+1}=\operatorname{argmin}_{\mathrm{x} \in \mathcal{X}} \mu_{m}(\mathrm{x})-\beta_{m} \sigma_{m}(\mathrm{x})
$$

where $\sigma_{m}(\mathrm{x}):=k_{m}(\mathrm{x}, \mathrm{x})$ indicates the variance in prediction of performance function at $\mathrm{x}$ and $\beta_{m}$ is a constant specifying the considered confidence bound around the mean function $\mu_{m}$. This procedure stops at iteration $m_{\mathrm{BO}}$ either by reaching the maximum number of iterations, or by sampling around the same configuration of parameters with minimal cost more than three times. The output of the algorithm is the vector of controller gains with the best observed performance, $\mathrm{x}_{m_{\mathrm{BO}}^{*}}$.

One could also tune the gains of the two loops sequentially, alternating between the gains of the inner and the outer loop, utilizing $\mathrm{BO}$ for each step [33]. We do not pursue this direction further here, since the combined tuning approach introduced above is more likely to lead to a globally optimal solution.

\section{Numerical Experiments}

We now compare the system trajectories of the transfer function model from Section III corresponding to BO tuning, classical methods, and an exhaustive (grid) computation of the performance metric on a $280 \times 90 \times 100$ grid on $\mathcal{X}$. To apply Algorithm 1 we initialize with dataset $\mathcal{D}_{0}$ collected from random locations in $\mathcal{X}$. In each iteration, we solve the GP-LCB optimization problem 9 by calculating the objective function of 9 for the points of the grid and finding the point with the optimal cost; note that this process does not require data to be collected at all the grid points, only that the Gaussian process is evaluated at these points. Table III shows that the controller gains, as well as the performance resulting from the 

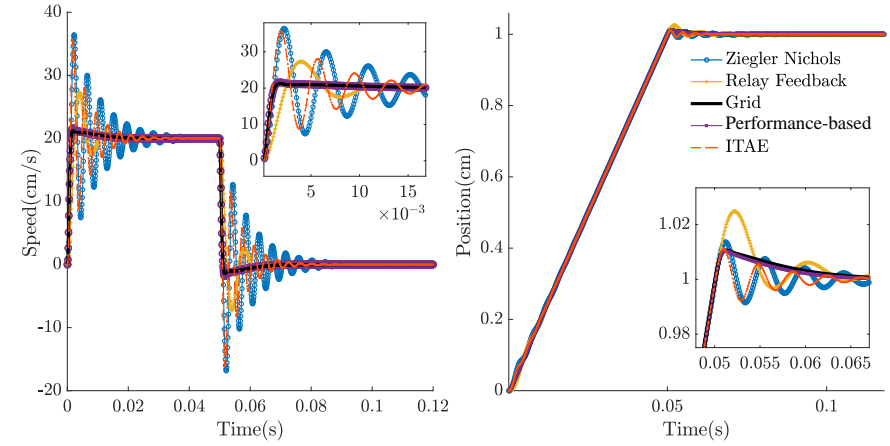

Figure 5: Response for different benchmark tuning methods

proposed method are almost the same as the performance of optimal grid point, i.e., the performance-tuning method finds the controller gains in the nearly flat region of performance around the optimal gains.

The closed-loop speed and position responses corresponding to benchmark tuning methods to the performance-based tuning responses, namely Zeigler-Nichols method, relay tuning, and ITAE in Figure 5 show significantly higher overshoot and oscillations compared to the performance-based tuning responses, which also matches the ideal response obtained by grid simulation. The position response tracks the input reference precisely, and the speed response has a slight overshoot in the acceptable range. Table [III shows the resulting control gains and corresponding performance. The result of the performance-based tuning is closest to the exhaustive evaluation results obtained on the grid while the gains obtained via standard tuning approaches (Zeigler-Nichols, relay tuning, and ITAE) are more aggressive. The number of training samples in $\mathcal{D}_{0}$ has a direct influence on the number of iterations needed to reach the stopping criterion that defines the converged controller gains. A more informed prior model requires less iterations during the optimization phase. We further performed a Monte Carlo simulation, where the algorithm was initialized with a different number of data points selected randomly from $\mathcal{X}$, and for each value of $m_{0}$ Algorithm 1 was executed 1000 times for a maximum number of 60 iterations. Based on the numerical experiments an initial data set $\mathcal{D}_{0}$ comprising 2050 different random configurations of parameters requires a tuning phase of 20 up to 50 iterations in total for convergence, which is a reasonable trade-off between the number of initial samples and tuning phase iterations. On a real system, each iteration takes up to 10 seconds. Since the performance metrics can be fully automated, and the initial exploration phase needs to be repeated only upon major changes in the system, the

Table III: Results of different tuning methods

\begin{tabular}{lcccc}
\hline Tuning method & $K_{\mathrm{p}}$ & $K_{\mathrm{v}}$ & $K_{\mathrm{i}}$ & $f$ \\
\hline Grid search (true optimal value) & 225 & 0.39 & 90 & 4557 \\
Ziegler Nichols & 392 & 0.18 & 510 & 28434 \\
ITAE criterion & 255 & 0.11 & 420 & 31163 \\
Relay Tuning & 115 & 0.05 & 130 & 23396 \\
Performance-based Tuning & 240 & 0.39 & 100 & 4586 \\
\hline
\end{tabular}
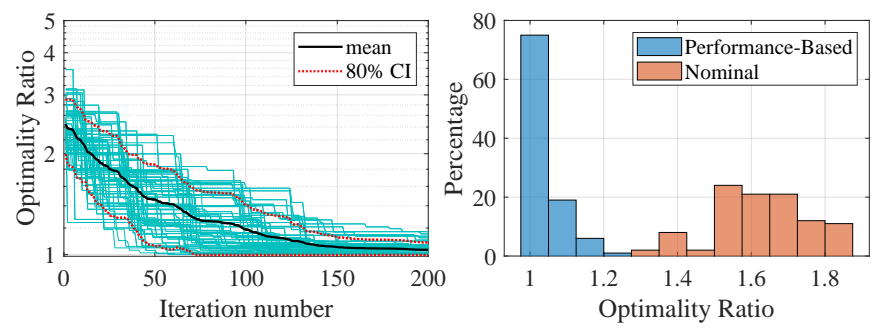

Figure 6: Results of Monte Carlo experiments - numerical verification for robustness with respect to changes in system parameters and initial set

proposed tuning method can be efficiently implemented.

The numerical experiments provide the range of stability in the controller gains. If the stability range is unknown, and if unstable parameters cannot be detected in advance, the application in reality might bring failures in the system. The method applied in practice is not influenced by the model or by the optimal parameters found in numerical experiments, as it provides the true optima according to the corresponding convergence criteria.

\section{Robustness Analysis}

In order to evaluate the robustness of the proposed method with respect to variations in all system parameters and different initial sets, we perform an extensive Monte Carlo analysis (see Figure 6. We perturb the physical parameters of the system randomly up to $5 \%$, and initialize each experiment with a random initial set of 30 random samples. Algorithm 1 is applied with fixed number of iterations to obtain the control gains. We compute the true optimal gains for each of the 100 experiments using particle swarm optimization. The results are compared by means of optimality ratio, defined as the performance of obtained control gains using Algorithm 1 normalized by the true optimal performance value. Figure 6 (left) shows that the performance improves iteratively and converges to the optimal value. Figure 6 (right) shows that the performance corresponding to the final (tuned) controller parameters is considerably improved compared to the performance corresponding to nominal values.

\section{Performance-Based Controller Tuning: EXPERIMENTAL RESULTS}

The linear motion system (stage) used to validate the proposed tuning method consists of a linear axis, a permanent magnet AC motor with a servo drive, and a Programmable Logic Controller (PLC), as shown in Figure 1 The system is equipped with a linear encoder with a precision of $1 \mu \mathrm{m} / \mathrm{m}$ and sampling time of $1 \mathrm{~ms}$ used for measuring the actual position and speed of the stage. The input voltage to the motor is provided based on the voltage reference signal from a Sigmatek S-DIAS PLC. Since the bandwidth of the current controller is above $1500 \mathrm{~Hz}$ and the corresponding bandwidth of the position controller does not exceed $400 \mathrm{~Hz}$, the current closed-loop can be well approximated with a constant gain. The proportional controller gains $K_{\mathrm{v}}$ and $K_{\mathrm{p}}$ of the cascade 
control loop are tuned, and the integral time constant $T_{\mathrm{n}}$ is tuned instead of the the integral gain $K_{\mathrm{i}}$.

For the joint tuning of the control parameters, the input setpoints (position, speed, acceleration, deceleration) were set identical for each run of the system. During the experiments, acceleration and deceleration values were kept high to simulate step input and step response in both position and speed control modes, and the speed was set at $20 \frac{\mathrm{cm}}{\mathrm{s}}$. The range of the three control parameters was limited following the numerical experiments to ensure that the system is always in a stable mode: $K_{\mathrm{p}} \in(0,65000], K_{\mathrm{v}} \in(0,7000], T_{\mathrm{n}} \in(1000,40000]$.

The position reference trajectory was a bi-directional step response, where the nut moves from 0 to $50 \mathrm{~cm}$, remains there for $10 \mathrm{~s}$, and returns to position 0 . In this case, input position and speed are set to $5 \mathrm{~cm}$ and $100 \frac{\mathrm{cm}}{\mathrm{s}}$, respectively. The modified weights used in the experimental implementation of the performance-based tuning are provided in Table IV. The termination criterion for tuning in practice was based on either achieving a predefined number of iterations, or reaching a minimum within a defined threshold and sampling at this candidate configuration for at least 3 iterations.

\section{A. Experimental Results: Performance and Robustness}

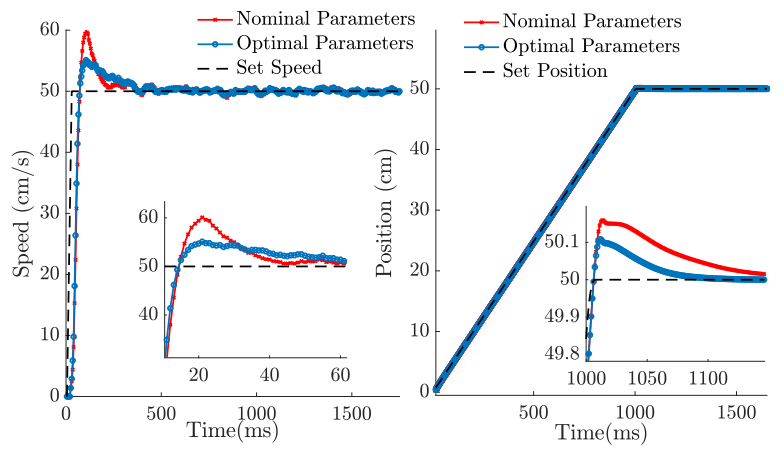

Figure 7: Position and speed responses comparing tracking traces of the system optimized with performance-based BO tuning and traces corresponding to the nominal parameters optimized by manual tuning. The insets show the initial overshoot obtained with each method.

Initially, 49 samples were obtained for different values of $K_{\mathrm{p}}, K_{\mathrm{v}}$ and $T_{\mathrm{n}}$. The required performance metrics were extracted from each of the data sets and the corresponding cost was calculated for each configuration of gains. Tuning the parameters using these training data for computing priors results

\begin{tabular}{lccc}
\hline \multicolumn{2}{c}{ position } & \multicolumn{2}{c}{ speed } \\
\hline$F_{i}^{(\mathrm{p})}$ & $\gamma^{(\mathrm{p})}$ & $F_{i}^{(\mathrm{s})}$ & $\gamma^{(\mathrm{s})}$ \\
\hline$T_{90}^{(\mathrm{p})}$ & $2 \cdot 10^{1}$ & $T_{90}^{(\mathrm{s})}$ & $2 \cdot 10^{1}$ \\
$h^{(\mathrm{p})}$ & $5 \cdot 10^{4}$ & $h^{(\mathrm{s})}$ & $10^{3}$ \\
$\left\|e^{(\mathrm{p})}\right\|_{\infty}$ & $5 \cdot 10^{4}$ & $e_{\mathrm{ITAE}}^{(\mathrm{s})}$ & $2.5 \cdot 10^{5}$ \\
$e_{0}^{(\mathrm{p})}$ & $10^{5}$ & $e_{\mathrm{s}}^{(\mathrm{s})}$ & $5 \cdot 10^{2}$ \\
& & $\bar{h}^{(\mathrm{s})}$ & $2 \cdot 10^{3}$ \\
\hline
\end{tabular}

Table IV: Weights in the cost function in convergence after 18 iterations. The optimal point is reached after repeated sampling near the optimum of the cost. The optimal values for the position and speed controller obtained are $K_{\mathrm{p}}=44500, K_{\mathrm{v}}=4000$ and $T_{\mathrm{n}}=12500$. The system response in position control mode using the above controller configuration for set position of $50 \mathrm{~cm}$ and set speed of $50 \frac{\mathrm{cm}}{\mathrm{s}}$ is shown in Figure 7. The negligible oscillation on the response is due to the amplified noisy resolver feedback, observed also in the nominal configuration. The speed response in the position control mode shown in Figure 7 also has an overshoot of $14 \%$ which is higher than the speed control mode as expected but is within an acceptable range with a maximum speed of $57.5 \frac{\mathrm{cm}}{\mathrm{s}}$. This is also in part due to the noise of the system. The position response steady state error is significantly smaller as compared to that in nominal mode, and the overshoot in position is less than $0.2 \mathrm{~cm}$ with very fast settling so there is no tracking delay or error. Performance-based tuning achieves better tracking and lower overshoot for both speed and position modes, and faster settling time for the more important position control mode. The results are summarized in Table [V], where it can be seen that the optimized parameters have reached higher values than the parameters from nominal tuning for the same load.

Because of the large number of samples in the training phase, the cost evolution during the $\mathrm{BO}$ iterations starts from a very low estimated cost, but with high uncertainty, and moves to higher cost, where the uncertainty is reduced after 10 iterations, as shown in Figure 8. Looking at the current minimal cost, it can be seen that the $\mathrm{BO}$-based tuning reaches a low-uncertainty cost which is very close to the converged results already at iteration 5 . The algorithm converges after 18 iterations, according to the specified somewhat conservative termination criterion.

We have further tested the robustness of the tuning approach by providing reference trajectories with different profiles. Figure 9 shows that the performance achieved with the BO tuning exceeds the nominal performance when there are fast changes in the speed, as in the top panels, and achieves better tracking of the references. The speed undershoot is significantly reduced, and better position stability is achieved at standstill, which is an important performance requirement for linear motion systems. For the trajectories shown in the bottom panels of Figure 9 the performance is virtually the same as nominal.

Standard tuning methods such as the Ziegler-Nichols rule or relay tuning show excellent performance for disturbance rejection objectives. We have evaluated the BO-based controller parameters with respect to disturbance rejection. Impulsive disturbance forces were applied during the operation in the

\begin{tabular}{ccccccc}
\hline & $m_{\mathrm{BO}}$ & $\begin{array}{c}K_{\mathrm{p}} \\
\times 10^{2}\end{array}$ & $\begin{array}{c}T_{\mathrm{n}} \\
\times 10^{2}\end{array}$ & $\begin{array}{c}K_{\mathrm{v}} \\
\times 10^{2}\end{array}$ & $\begin{array}{c}f^{(\mathrm{p})} \\
\times 10^{3}\end{array}$ & $\begin{array}{c}f^{(\mathrm{s})} \\
\times 10^{3}\end{array}$ \\
\hline Nominal (no load) & - & 200 & 100 & 25 & 523 & 271 \\
Perf.-based (no load) & 67 & 445 & 125 & 40 & 401.9 & 224.9 \\
Perf.-based (extra load) & 43 & 650 & 130 & 42.5 & 254.2 & 281.8 \\
\hline
\end{tabular}

Table V: Summary of all tuning results on the linear drive 


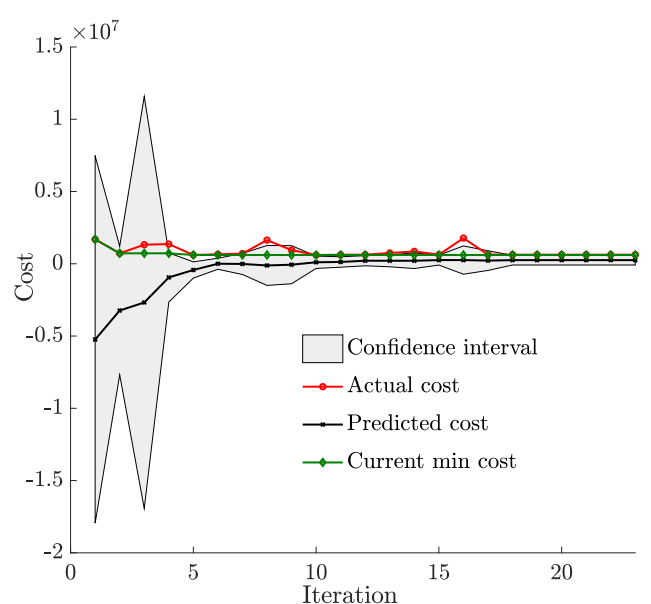

Figure 8: Predicted, actual observed cost, and associated confidence interval at 3 standard deviations for performancebased BO tuning on the system.

direction of movement of the system with additional load. The resulting response is shown in Figure 10, and compared with the system's nominal performance when subjected to the same type of disturbance. The response of the system shows a quick recovery following the disturbance, as shown in Figure 10 . Even though the applied disturbance has significantly higher magnitude than in the nominal case, the observed recovery is faster for the performance-based tuned controller gains. This is due to the performance metric term that corresponds to tracking accuracy and position stability.

\section{B. Discussion}

The proposed performance-based BO tuning thus offers a trade-off between grid based search, and heuristic-based methods. The method requires more iterations to converge

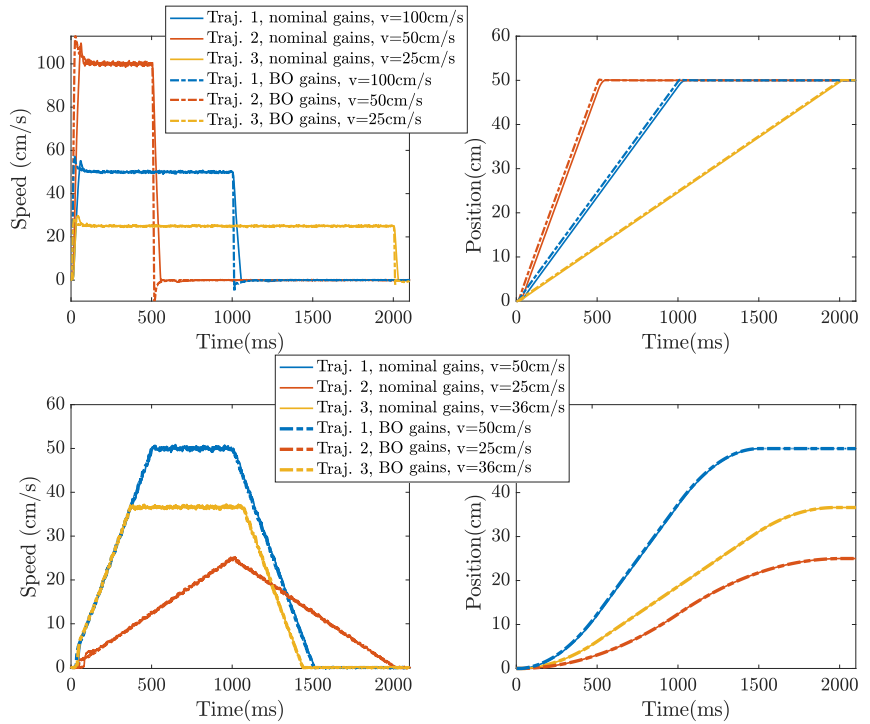

Figure 9: Position and speed responses comparing tracking with different trajectories. Upper panels: Position and speed responses for different step signals; Bottom panels: Trajectories with varying speed setpoints.

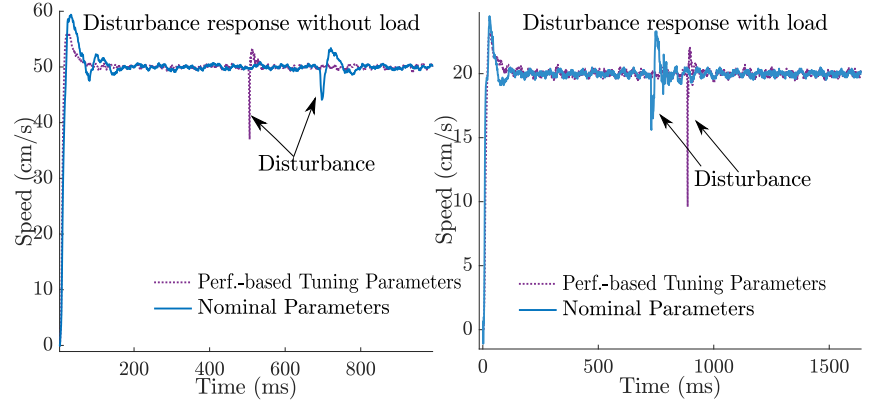

Figure 10: Speed responses of the system without and additional load, comparing disturbance response of the nominal controller and the BO-optimized controller.

compared to relay feedback tuning, but finds an optimum in the specified performance objective. It provides disturbance rejection and superior performance for various trajectories, irrespective of the trajectory used in training, and is easily adapted for different loads. The duration of the tuning procedure is acceptable in practice and does not exceed 120 iterations with a total duration of up to one hour. A potential drawback is the required system knowledge to ensure stability. Currently, this is achieved by limiting the optimization range to avoid destabilizing values of the parameters. The ranges are derived either based on the model of the system or based on expert knowledge from operating the system. When the ranges are not a priori known, and for the full automation of the method, it would be useful to ensure a safety mechanism in the performance-based tuning procedure. This could be achieved either through the addition of the safety constraints such as Gaussian processes in the BO algorithm or through the modification of the acquisition function to account for the probability of constraint violation [18]. Another possibility is to detect experimentally when the system is approaching critical regime and to include a safety penalty in the cost [25].

The proposed tuning method automates the optimization of a controller. The achieved performance is limited by the worst point encountered during the tuning procedure. The method can be implemented in an adaptive scheme by continuously monitoring the performance and re-optimizing upon deviations from the optimum. Several data-driven approaches for adaptive control have been recently proposed, such as [34], [35] and [36], with theoretical guarantees for stability of the proposed control algorithms. The proposed performance-based tuning method thus can be used for data-driven adaptive control, provided a suitable safe $\mathrm{BO}$ algorithm is put in place.

\section{CONCLUSION AND OUTLOOK}

We have presented a data-driven approach for cascade controller tuning, where we model the performance of a closed-loop system as a function of controller gains. We apply a Bayesian optimization approach to derive controller gains with optimal performance. The performance was first evaluated in a simulation for a ball-screw linear axial system, and compared to classical tuning approaches and the computed optimal performance on a grid. The experimental validation of the proposed method shows that it enables fast and standard- 
ized tuning, with a performance superior to other autotuning approaches. It allows easy adaptation of the controller parameters upon changes in the load or the system's mechanical configuration. Extending the method with automatic detection of instabilities will further increase its flexibility and potential for practical use.

\section{Appendix: Gaussian Processes Regression}

A Gaussian process (GP) is a collection of random variables where each of its finite subsets is jointly Gaussian [27]. The GP is uniquely characterized by the mean function, $\mu: \mathcal{X} \rightarrow$ $\mathbb{R}$, and the covariance/kernel function, $k: \mathcal{X} \times \mathcal{X} \rightarrow \mathbb{R}$, where $\mathcal{X} \subseteq \mathbb{R}^{d}$ is the set of location indices. The corresponding GP is denoted by $\mathcal{G P}(\mu, k)$. Gaussian processes provide flexible models for Bayesian learning by introducing prior distributions over the space of functions defined on $\mathcal{X}$. Due to the favorable properties of the Gaussian distributions, the marginal and the conditional mean and variance can be computed on any finite set of locations in a closed form. Let $f: \mathcal{X} \rightarrow \mathbb{R}$ be an unknown function, $\mathcal{G P}(0, k)$ be the prior distribution for estimating $f$, and noisy measurements at locations $\mathrm{x}_{1}, \ldots, \mathrm{x}_{m}$ be respectively given as $\mathrm{y}_{1}, \ldots, \mathrm{y}_{m}$. In other words, we know that $f \sim \mathcal{G P}(0, k)$ and $\mathrm{y}_{i}=f\left(\mathrm{x}_{i}\right)+w_{i}$, for $i=1, \ldots, m$, where $w_{i}$ is the measurement noise with zero mean Gaussian distribution $\mathcal{N}\left(0, \sigma_{w}^{2}\right)$. Since there is no additional information about the latent function $f$, we can set the mean of the GP prior to zero. Let $\mathrm{x} \in \mathcal{X}$ be a new location where the corresponding measurement is not provided. Since the joint distribution of the measurements data is Gaussian with a given mean and a given covariance, one can predict the value of measurement at the new location $\mathrm{x}$ using Bayes rule. First, we define $\mathrm{X}_{m}$ and $\mathrm{y}_{m}$ respectively as $\mathrm{X}_{m}:=\left[\mathrm{x}_{1}, \mathrm{x}_{2}, \ldots, \mathrm{x}_{m}\right] \in \mathbb{R}^{n \times m}$ and $\mathrm{y}_{m}:=\left[y_{1}, y_{2}, \ldots, y_{m}\right]^{\top} \in \mathbb{R}^{m}$. Also, we define the Gram matrix $\mathrm{K}_{\mathrm{X}_{m} \mathrm{X}_{m}} \in \mathbb{R}^{m \times m}$ such that its element at the $i^{\text {th }}$ row and the $j^{\text {th }}$ column is given by $k\left(\mathrm{x}_{i}, \mathrm{x}_{j}\right)$. Consequently, due to $f \sim \mathcal{G P}(0, k)$, we know that $\mathrm{y}_{m} \sim \mathcal{N}\left(\mathbf{0}, \mathrm{K}_{\mathrm{X}_{m} \mathrm{X}_{m}}+\sigma_{w}^{2} \mathbb{I}\right)$, where II denotes the identity matrix. The joint distribution of the training data $\left\{\left(\mathrm{x}_{i}, y_{i}\right)\right\}_{i=1}^{m}$ with the new data point $\left(\mathrm{x}, y_{\mathrm{x}}\right)$, where $y_{\mathrm{x}}:=f(\mathrm{x})$ is the unknown label for location $\mathrm{x}$, can be calculated as

$$
\left[\begin{array}{c}
\mathrm{y}_{m} \\
y_{\mathrm{x}}
\end{array}\right] \sim \mathcal{N}\left(0,\left[\begin{array}{cc}
\mathrm{K}_{\mathrm{X}_{m} \mathrm{X}_{m}}+\sigma_{w}^{2} \mathbb{I} & \mathrm{k}_{\mathrm{xX}_{m}}^{\top} \\
\mathrm{k}_{\mathrm{xX}_{m}} & k_{\mathrm{xx}}
\end{array}\right]\right),
$$

where $\mathrm{k}_{\mathrm{xX}_{m}} \in \mathbb{R}^{m}$ is the vector such that its $i^{\text {th }}$ element is given by the kernel as $k\left(\mathrm{x}, \mathrm{x}_{i}\right)$, for any $i=1, \ldots, m$, and $k_{\mathrm{xx}}:=k(\mathrm{x}, \mathrm{x})$. The posterior distribution of $y_{\mathrm{x}} \mid \mathrm{X}_{m}, \mathrm{y}_{m}$ is a Gaussian distribution as $y_{\mathrm{x}} \mid \mathrm{X}_{m}, \mathrm{y}_{m} \sim \mathcal{N}\left(\mu_{m}(\mathrm{x}), \sigma_{m}(\mathrm{x})\right)$, where the mean of prediction, $\mu_{m}(\mathrm{x})$, and the corresponding covariance, $\sigma_{m}(\mathrm{x})$, are given as

$$
\begin{aligned}
\mu_{m}(\mathrm{x}) & :=\mathrm{k}_{\mathrm{x}_{m}}^{\top}\left(\mathrm{K}_{\mathrm{X}_{m} \mathrm{X}_{m}}+\sigma_{w}^{2} \mathbb{I}\right)^{-1} \mathrm{y}_{m} \\
\sigma_{m}(\mathrm{x}) & :=k_{\mathrm{xx}}-\mathrm{k}_{\mathrm{xX}_{m}}^{\top}\left(\mathrm{K}_{\mathrm{X}_{m} \mathrm{X}_{m}}+\sigma_{w}^{2} \mathbb{I}\right)^{-1} \mathrm{k}_{\mathrm{x}_{m}} .
\end{aligned}
$$

The posterior mean $\mu_{m}(\mathrm{x})$ is a nonlinear function predicting the value of $f$ at location $\mathrm{x}$ with an uncertainty described by $\sigma_{m}(\mathrm{x})$. Accordingly, this is a nonlinear non-parametric regression method called Gaussian process regression (GPR). The hyperparameters of the kernel $\sigma_{f}, \mathrm{~L}$ and $\sigma_{w}$ collected in a vector $\theta \in \Theta$ can be estimated by minimizing the negative log marginal log-likelihood of the joint distribution of the training data, i.e.,

$$
\hat{\theta}:=\operatorname{argmin}_{\theta \in \Theta}-\log p\left(\mathrm{y}_{m} \mid \mathrm{X}_{m}, \theta\right),
$$

where $p\left(\mathrm{y}_{m} \mid \mathrm{X}_{m}, \theta\right)$ is the probability density function of the labels or measurements acquired at locations $\left\{\mathrm{x}_{i}\right\}_{i=1}^{m}$.

\section{REFERENCES}

[1] K. J. Aström and R. M. Murray, Feedback Systems: An Introduction for Scientists and Engineers. USA: Princeton University Press, 2008.

[2] C. Kessler, "Das symmetrische Optimum," Regelungstechnik, vol. 6, pp. $395-400,432-436,1958$.

[3] S. Preitl and R.-E. Precup, "An extension of tuning relations after symmetrical optimum method for PI and PID controllers," Automatica, vol. 35, no. 10 , pp. 1731 - 1736, 1999.

[4] A. Karimi and C. Kammer, "A data-driven approach to robust control of multivariable systems by convex optimization," Automatica, vol. 85, pp. $227-233,2017$.

[5] W. G. da Silva, P. P. Acarnley, and J. W. Finch, "Application of genetic algorithms to the online tuning of electric drive speed controllers," IEEE Transactions on Industrial Electronics, vol. 47, no. 1, pp. 217-219, 2000.

[6] Z. Qi, Q. Shi, and H. Zhang, "Tuning of digital PID controllers using particle swarm optimization algorithm for a CAN-based DC motor subject to stochastic delays," IEEE Transactions on Industrial Electronics, vol. 67, no. 7, pp. 5637-5646, 2020.

[7] J. Ziegler and N. Nichols, "Optimum settings for automatic controllers," Transactions of the ASME, vol. 64, pp. 759-768, 1942.

[8] C. Hang, K. Åström, and Q. Wang, "Relay feedback auto-tuning of process controllers-a tutorial review," Journal of process control, vol. 12, no. 1, pp. 143-162, 2002

[9] R. Kumar, S. Singla, and V. Chopra, "Comparison among some well known control schemes with different tuning methods," Journal of Applied Research and Technology, vol. 13, no. 3, pp. 409 - 415, 2015.

[10] M. Campi, A. Lecchini, and S. Savaresi, "Virtual reference feedback tuning: a direct method for the design of feedback controllers," Automatica, vol. 38, no. 8, pp. 1337 - 1346, 2002.

[11] M. C. Campi and S. M. Savaresi, "Direct nonlinear control design: the virtual reference feedback tuning (VRFT) approach," IEEE Transactions on Automatic Control, vol. 51, no. 1, pp. 14-27, 2006.

[12] C. Novara and S. Formentin, "Data-driven inversion-based control of nonlinear systems with guaranteed closed-loop stability," IEEE Transactions on Automatic Control, vol. 63, no. 4, pp. 1147-1154, 2018

[13] S. Formentin, D. Piga, R. Toth, and S. M. Savaresi, "Direct learning of LPV controllers from data," Automatica, vol. 65, pp. 98 - 110, 2016.

[14] H. Prochazka, M. Gevers, B. D. O. Anderson, and C. Ferrera, "Iterative feedback tuning for robust controller design and optimization," in Conference on Decision and Control, pp. 3602-3607, 2005.

[15] M. Rădac, R. Precup, E. M. Petriu, and S. Preitl, "Iterative data-driven tuning of controllers for nonlinear systems with constraints," IEEE Transactions on Industrial Electronics, vol. 61, DOI 10.1109/TIE.2014.2300068 no. 11, pp. 6360-6368, Nov. 2014.

[16] A. S. Bazanella, L. Campestrini, and D. Eckhard, Data-Driven Controller Design: The $\mathrm{H}_{2}$ Approach. Springer Publishing Company, Incorporated, 2014.

[17] S. Bansal, R. Calandra, T. Xiao, S. Levine, and C. J. Tomiin, "Goaldriven dynamics learning via Bayesian optimization," in Conference on Decision and Control, pp. 5168-5173, 2017.

[18] M. Maier, R. Zwicker, M. Akbari, A. Rupenyan, and K. Wegener, "Bayesian optimization for autonomous process set-up in turning," CIRP Journal of Manufacturing Science and Technology, vol. 26, DOI https://doi.org/10.1016/j.cirpj.2019.04.005 pp. 81 - 87, 2019.

[19] O. Andersson, M. Wzorek, P. Rudol, and P. Doherty, "Model-predictive control with stochastic collision avoidance using Bayesian policy optimization," in 2016 IEEE International Conference on Robotics and Automation (ICRA), DOI 10.1109/ICRA.2016.7487661 pp. 4597-4604, May. 2016.

[20] F. Berkenkamp, A. P. Schoellig, and A. Krause, "Safe controller optimization for quadrotors with Gaussian processes," 2016 IEEE International Conference on Robotics and Automation, pp. 491 - 496, 2016.

[21] M. Neumann-Brosig, A. Marco, D. Schwarzmann, and S. Trimpe, "Dataefficient auto-tuning with Bayesian optimization: An industrial control study," IEEE Transactions on Control Systems Technology, 2018. 
[22] M. Khosravi, A. Eichler, N. Schmid, P. Heer, and R. S. Smith, "Controller tuning by Bayesian optimization an application to a heat pump," in European Control Conference, pp. 1467-1472. IEEE, 2019.

[23] M. Khosravi, N. Schmid, A. Eichler, P. Heer, and R. S. Smith, "Machine learning-based modeling and controller tuning of a heat pump," in Journal of Physics: Conference Series, vol. 1343, no. 1, p. 012065. IOP Publishing, 2019.

[24] F. Berkenkamp, A. Krause, and A. P. Schoellig, "Bayesian optimization with safety constraints: Safe and automatic parameter tuning in robotics," arXiv:1602.0445, 2016.

[25] C. König, M. Khosravi, M. Maier, R. S. Smith, A. Rupenyan, and J. Lygeros, "Safety-Aware Cascade Controller Tuning Using Constrained Bayesian Optimization," arXiv e-prints, p. arXiv:2010.15211, Oct. 2020

[26] Y. Altintas, A. Verl, C. Brecher, L. Uriarte, and G. Pritschow, "Machine tool feed drives," CIRP annals, vol. 60, no. 2, pp. 779-796, 2011.

[27] C. E. Rasmussen and C. K. I. Williams, Gaussian Processes for Machine Learning. MIT Press, 2006.

[28] N. Srinivas, A. Krause, S. M. Kakade, and M. W. Seeger, "Informationtheoretic regret bounds for Gaussian process optimization in the bandit setting," IEEE Transactions on Information Theory, vol. 58, no. 5, pp. $3250-3265,2012$.

[29] K. K. Varanasi and S. A. Nayfeh, "The dynamics of lead-screw drives: Low-order modeling and experiments," Journal of Dynamic Systems, Measurement, and Control, ASME, vol. 126, pp. 388 - 398, Jun. 2004.

[30] R. Qian, M. Luo, J. Zhao, and T. Li, "Novel sliding mode control for ball screw servo system," in MATEC Web of conferences, 7th International Conference on Mechanical, Industrial, and Manufacturing Technologies, ser. 03007, vol. 54, 2016.

[31] K. J. Åström, T. Hägglund, C. C. Hang, and W. K. Ho, "Automatic tuning and adaptation for PID controllers-a survey," Control Engineering Practice, vol. 1, no. 4, pp. 699-714, 1993.

[32] M. D. McKay, R. J. Beckman, and W. J. Conover, "A comparison of three methods for selecting values of input variables in the analysis of output from a computer code," Technometrics, vol. 42, no. 1, pp. 55-61, 2000.

[33] M. Khosravi, V. Behrunani, R. S. Smith, A. Rupenyan, and J. Lygeros, "Cascade control: Data-driven tuning approach based on Bayesian optimization," 2020 IFAC World Congress (arXiv:2005.03970), 2020.

[34] T. Yang, N. Sun, H. Chen, and Y. Fang, "Neural network-based adaptive antiswing control of an underactuated ship-mounted crane with roll motions and input dead zones," IEEE Transactions on Neural Networks and Learning Systems, vol. 31, no. 3, pp. 901-914, 2020.

[35] N. Sun, D. Liang, Y. Wu, Y. Chen, Y. Qin, and Y. Fang, "Adaptive control for pneumatic artificial muscle systems with parametric uncertainties and unidirectional input constraints," IEEE Transactions on Industrial Informatics, vol. 16, no. 2, pp. 969-979, 2020.

[36] G. Chowdhary, H. A. Kingravi, J. P. How, and P. A. Vela, "Bayesian nonparametric adaptive control using gaussian processes," IEEE Transactions on Neural Networks and Learning Systems, vol. 26, no. 3, pp. 537-550, 2015.

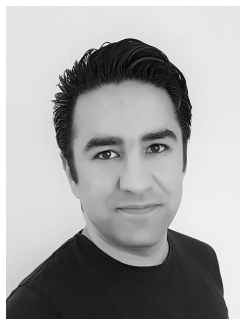

Mohammad Khosravi received a B.Sc. in electrical engineering and a B.Sc. in mathematical sciences from Sharif University of Technology, Tehran, Iran, in 2011. He obtained a postgraduate diploma in mathematics from ICTP, Trieste, Italy, in 2012. He was a research assistant in BioMath group of Institute for Research in Fundamental Sciences, Tehran, Iran, in 2012-2014. He received his M.A.Sc. degree in electrical and computer engineering from Concordia University, Montreal, Canada, in 2016. Currently, he is a doctoral student at Automatic Control Laboratory, ETH Zürich. His research interest is the theory of data-driven modeling and decisionmaking, and their applications in energy and industrial systems.

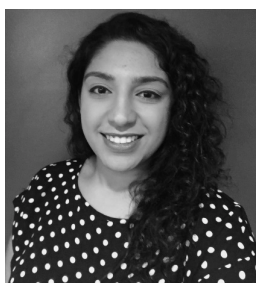

Varsha N. Behrunani received the B. Sc. degree in electrical engineering from R.I.T University in Dubai, UAE in 2016 and is finishing her MSc in electrical engineering with a focus in advanced systems and control from ETH Zürich since September 2018, following several industry appointments in electrical engineering. She is currently working as a research and teaching assistant at the high power electronics lab at ETH Zürich. Her research interests are focused on data-based control, distributed control, and

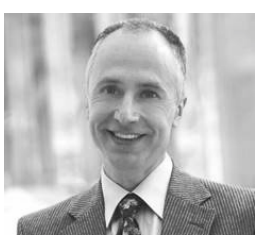

Piotr Myszkorowski received the M.Sc. degree in electrical engineering and Ph.D. degree from the Technical University of Lodz, Poland, in 1979 and 1988 respectively and a M.Sc. degree in mathematics from the University of Lodz, Poland, in 1983. After assistant and assistant professor appointments with the Technical University of Lodz and a Visiting Professor appointment at INRIA, Sophia Antipolis, France, he joined the EPFL as Postdoctoral Researcher and Lecturer between 1992 and 1999. He was subsequently the technical manager at the Swiss office of Baumüller Nürnberg $\mathrm{GmbH}$, and since 2009 he is technical manager at Sigmatek Schweiz AG. His research interests include optimal and robust control of both linear and nonlinear systems.

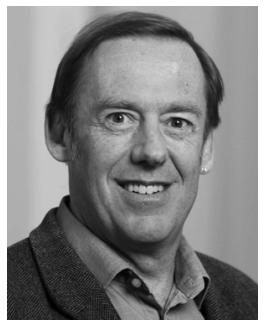

Roy S. Smith (F11) received the bachelor's degree from the University of Canterbury, Christchurch, New Zealand, in 1980, and the Ph.D. degree from the California Institute of Technology, Pasadena, CA, USA, in 1990. From 1990 to 2010, he was a Faculty of the University of California at Santa Barbara, USA. In 2011, he joined ETH Zürich, Switzerland, where he is currently a professor of electrical engineering. He has been a long-time Consultant to the NASA Jet Propulsion Laboratory, Pasadena, CA, USA and has industrial experience in automotive control and power system design. His current research interests include the modeling, identification, and control of uncertain systems, especially chemical processes, flexible structure vibration, spacecraft and vehicle formations, aerodynamic control of kites, buildings. Prof. Smith is an Associate Fellow of the American Institute of Aeronautics and Astronautics and a member of the Society for Industrial and Applied Mathematics.

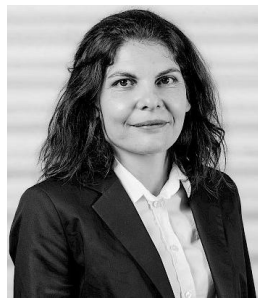

Alisa Rupenyan (M18) received the BSc in Engineering physics and the MSc degree in Laser physics from the University of Sofia, Sofia, Bulgaria, in 2004 and 2005, and the Ph.D. degree from the Department of Physics and Astronomy of Vrije Universiteit Amsterdam, Amsterdam, The Netherlands. Between 2011-2014 she was a postdoctoral fellow at ETH Zürich 2011, Switzerland, and between 2014-2018 a lead scientist in a robotic start-up. She is currently a group leader in automation at inspire, the technology transfer unit at ETH Zürich, and a senior scientist and $\mathrm{PI}$ at the Automatic Control Laboratory there. Her research interests are on the intersection between machine learning, control, and optimization for industrial applications and robotics, especially in Bayesian methods applied for optimization and control, and learning-based control. Dr. Rupenyan is a member of the IEEE Robotics and Automation Society where she is on the Technical committee for Model-based optimization in robotics, and a member of the IFAC industry committee.

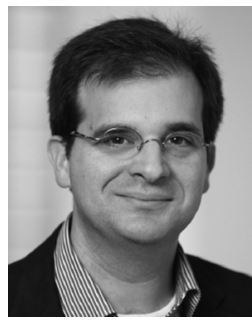

John Lygeros (F11) received the B.Eng. degree in electrical engineering and the M.Sc. degree in systems control from the Imperial College of Science Technology and Medicine, London, U.K., in 1990 and 1991, respectively, and the Ph.D. degree from the Electrical Engineering and Computer Sciences Department, University of California, Berkeley, Berkeley, CA, USA, in 1996. After postdoctoral researcher appointments at M.I.T. and U.C. Berkeley, in 2000, he joined the Department of Engineering, University of Cambridge, U.K., as a Lecturer and Churchill College as a Fellow. In July 2006, he joined the Automatic Control Laboratory at ETH Zürich, first as an Associate Professor, and in January 2010, as a Full Professor. Since 2009, he has been serving as the Head of the Automatic Control Laboratory. His research interests include modeling, analysis, and control of hierarchical, hybrid, and stochastic systems, with applications to biochemical networks, automated highway systems, air traffic management, power grids and camera networks. Prof. Lygeros is a member of the IET and the Technical Chamber of Greece; since 2013, he has been serving as the Treasurer of the International Federation of Automatic Control and a member of the IFAC Council. 